\title{
Could Parkinson's disease follow intra-uterine influenza?: a speculative hypothesis
}

\author{
CHRISTOPHER MATTOCK, MICHAEL MARMOT, GERALD STERN
}

From the Departments of Community Medicine and Neurology, University College and Middlesex School of Medicine, London, UK

Summary Patients with idiopathic Parkinson's disease do not appear to be distributed smoothly with respect to year of birth. Individuals born within the years 1892, 1904, 1909, 1918, 1919 and 1929 appear to have had an increased risk of developing idiopathic Parkinson's disease in later life. These years are close to those of the influenza pandemics of the period 1890-1930. The estimated risk of an individual developing idiopathic Parkinson's disease shows a significant correlation with the crude influenza mortality for the year of his birth, within the range 1900 to 1930 . It is suggested that intra-uterine influenza may be cytotoxic to the developing foetal substantia nigra, and that an affected individual may be born without evident disability but with limited striatal neurochemical reserves and a reduced nigral cell count. In later life normal cellular involution with ageing or exposure to environmental neurotoxic factors may further erode these reserves to a level where the substantia nigra fails and idiopathic Parkinson's disease becomes clinically apparent.

The absence of significant genetic factors in the aetiology of idiopathic Parkinson's disease has led to scrutiny of possible environmental factors; the neurochemical evidence that marked and presumably slowly developing deficiency of striatal dopamine precedes clinical recognition of the disease suggests that the cause may be an early event. Poskanzer and Schwab, ${ }^{1}$ on the basis of a cohort analysis, proposed that an exposure, possibly viral, could lead to idiopathic disease in later life, but this has not been generally accepted. We found that patients with idiopathic Parkinson's disease attending our clinic were clustered by year of birth. To determine whether birth clustering was a more general feature of idiopathic Parkinson's disease, people previously identified in two unrelated surveys of Parkinson's disease, in 1971 and 1978, were studied. If clustering occurs it would be compatible with an in utero or neonatal influence upon the subsequent risk of developing idiopathic Parkinson's disease.

\section{Patients and methods}

Two groups of Parkinsonian patients were studied. Group 1 (297 patients) were all those under treatment and well

Address for reprint requests: Dr Gerald Stern, Department of Neurology, Middlesex Hospital, Mortimer Street, London WIN 8AA.

Received 2 February 1988. Accepted 16 February 1988 enough to attend our idiopathic Parkinson's disease clinic in 1984. They were matched to 1485 controls attending other clinics in the same hospital. Group 2 (604 patients) came from two sources: 220 patients and $\mathbf{4 1 0}$ controls, identified by their general practitioners in 1971 as part of a study of the disease-specific mortality in idiopathic Parkinson's disease (Marmot - unpublished observations) and 384 patients and 384 controls identified by their general practitioners in 1978 as part of a study of possible causative factors in idiopathic Parkinson's disease. ${ }^{2}$

Matched controls were selected so that they were born within the same quinquennium (that is, 1900-1904, 1905-1909 etc) as the index patient. For each group and each calendar year the ratio of Parkinsonian to control births was calculated (P:C ratio), with allowance made for differing control allocations. This ratio was used to estimate the relative risk of each cohort developing idiopathic Parkinson's disease in later life. To avoid the misleading efiects of small numbers upon ratios, calendar years with fewer than four Parkinsonian births were excluded. Calendar years with a P:C ratio of 1.66 or greater were arbitrarily defined as "cluster years", and by inference individuals born in these years have an above average risk of developing idiopathic Parkinson's disease.

Dates of the infiuenza pandemies were taken from monographs on influenza. ${ }^{3-5}$ Figures for crude infiuenzal mortality rates for England and Wales (per 100,000 population) were obtained from the annual reports of the Registrar General. 67

\section{Resilts}

Years in which four or more Parkinsonian patients 753 


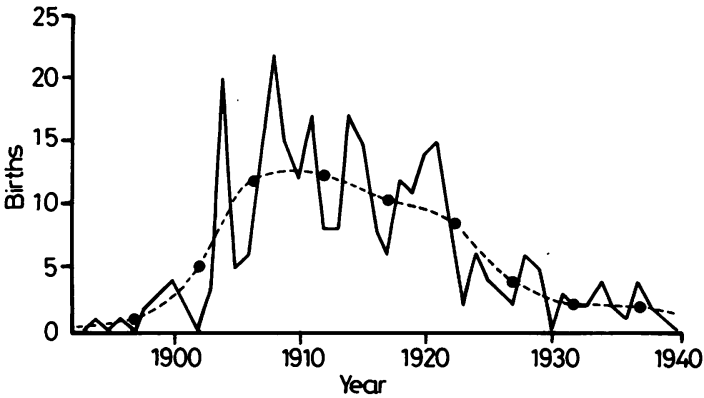

Fig 1 The actual distribution of Group 1 Parkinsonian births (solid lines) contrasted with an "expected" smoother curve drawn through the actual mean birth rate of Group 1 Parkinsonians for each quinquennium.

were born ranged from 1900 to 1937 in Group 1 and from 1881 to 1930 in Group 2; data were analysed for the overlapping period 1900 to 1930 .

Figure 1 suggests that Group 1 Parkinsonians are not distributed smoothly with respect to year of birth. The dashed line connects the mean birth rate of idiopathic Parkinson's disease patients for each quinquennium (the circles) and this curve provides an "expected" birth distribution for comparison, assuming no year to year fluctuations. Actual Group 1 Parkinsonian births differ significantly from this "expected" distribution, chi-square $61.6(\mathrm{p}<0.001)$, as they also do from the distribution of their matched controls, chi-square 86.0 ( $p<0.001)$. Group 2 Parkinsonians also differ significantly from their matched control distribution, chi-square 54.5 (p < 0.01) and from their "expected" curve.

Three "cluster years" occur in each group between 1900 and 1930, and table 1 provides a summary. The

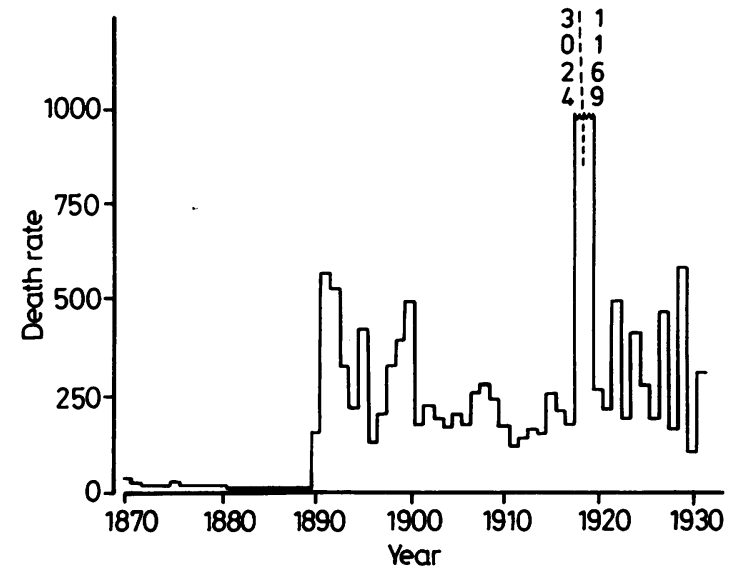

Fig 2 Influenzal mortality rate per million population for England and Wales 1870-1931. (Registrar-General). *(1918: 3024 deaths per million population) (1919: 1169 deaths per million population).

cluster years of each group coincide in 1918 and 1929. The probability of such a match ( 2 out of 3 ) within a 31 year span by chance alone is $0.039(\mathrm{p}<0.05)$. Thus a common factor in the two independent populations may underlie clustering.

As the two independent Groups 1 and 2 both show year to year fluctuations in Parkinsonian births and the major cluster years tend to coincide, the data were pooled for further analysis. These pooled data contain six years, 1892, 1904, 1909, 1918, 1919, and 1929, in which significantly more Parkinsonians, than controls were born when assessed at the $2.5 \%$ level, as summarised in table 2 . Four of these years coincide with years of influenza pandemics of 1892, 1918-1919

Table 1 Years in which a Parkinsonian:control birth ratio (P:C ratio) of greater than 1.66 was noted in group 1 and/or group 2

\begin{tabular}{|c|c|c|c|c|}
\hline \multirow[b]{2}{*}{ Year } & \multicolumn{2}{|l|}{ Group 1} & \multicolumn{2}{|l|}{ Group 2* } \\
\hline & Births $(P: C)$ & P:C Ratio & Births $(P: C)$ & P:C Ratio \\
\hline $\begin{array}{l}1904 \\
1918 \\
1919 \\
1929\end{array}$ & $\begin{array}{c}18: 4 \cdot 7 \\
12: 7 \cdot 0 \\
11: 10 \cdot 8 \\
5: 3\end{array}$ & $\begin{array}{l}3.83 \\
1.71 \\
1.02 \\
1.67\end{array}$ & $\begin{array}{c}32: 23 \\
9: 5 \\
14: 5 \\
5: 2\end{array}$ & $\begin{array}{l}1.39 \\
1.80 \\
2.80 \\
2.50\end{array}$ \\
\hline
\end{tabular}

*Clusters were also noted for group 2 in $1889(8: 4 \cdot 5)$ and 1892 (13:7).

Table 2 Years in which the Parkinsonian:control birth ratio (P:C ratio) was significantly high, for Groups 1 and 2 combined

\begin{tabular}{|c|c|c|c|c|}
\hline Year & Births (P:C) & Chi-square & p-value & Influenza pandemic \\
\hline $\begin{array}{l}1892 \\
1904 \\
1909 \\
1918 \\
1919 \\
1929\end{array}$ & $\begin{array}{l}13: 7 \cdot 0 \\
50: 27 \cdot 4 \\
41: 28 \cdot 8 \\
21: 12 \cdot 0 \\
25: 15 \cdot 8 \\
10: 5 \cdot 0\end{array}$ & $\begin{array}{r}5 \cdot 28 \\
19 \cdot 23 \\
5 \cdot 34 \\
6 \cdot 84 \\
5 \cdot 45 \\
5 \cdot 03\end{array}$ & $\begin{array}{l}<0.025 \\
<0.001 \\
<0.025 \\
<0.01 \\
<0.02 \\
<0.025\end{array}$ & $\begin{array}{l}+ \\
- \\
- \\
+ \\
+ \\
+\end{array}$ \\
\hline
\end{tabular}




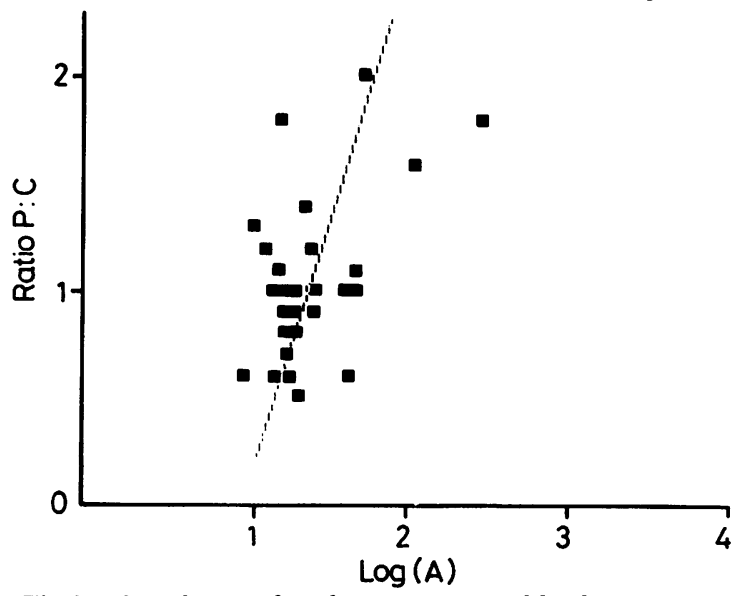

Fig 3 Correlation of Parkinsonian:control birth ratio (relative idiopathic Parkinson's disease risk) with the logarithm of the influenza mortality rate per 100,000 population in the year of birth $(\log A)$. Correlation coefficient $+0.503, p<0.005$.

and 1929. The probability of such a match (4 out of 6 ) in a 39 year span is $0.0044(p<0.005)$, suggesting a link between birth in an influenza pandemic year and the later emergence of idiopathic Parkinson's disease. This suggestion is further supported by the finding of a positive correlation between the $\mathrm{P}: \mathrm{C}$ ratio and influenzal deaths (per 100,000 population) in the years of birth 1900 to 1930 (correlation coefficient $+0.503, p<0.005$ ). As can be seen from fig 2 the effects of the influenza pandemics are to skew the mortality rates to the right, and therefore the correlation was reassessed substituting the logarithm of the annual influenzal death rate. This correlation (coefficient also $+0.503, \mathrm{p}<0.005$ ) is shown in fig 3 and the equation of the regression line is:

$$
\text { Relative IPD risk }=2 \cdot 4 \log (\mathrm{A})-2 \cdot 4
$$

(where $A$ is the annual influenzal death rate per 100,000 population in the year of birth).

When the pooled Parkinsonian data were analysed by month of birth there were excess Parkinsonian births in May (chi-square 3.9, p < 0.05) and a paucity of births in July (chi-square 4.6, p $<0.05$ ). When the calendar year was divided into three periods pooled Parkinsonian births were increased from March-June, reduced for July-October, and did not differ from matched control births for November-February, chi-square $8 \cdot 2, p<0.02$.

\section{Discussion}

The finding that the year of birth distribution for the two independent groups of Parkinsonian patients differs from that of matched controls confirms our initial impression and raises the possibility that birth clustering may be a general feature of idiopathic Parkinson's disease. This finding may of course be attributed to chance, but the pooled sample size of 901 patients and the estimates of probability suggest that chance alone is unlikely. Further, the technique employed to match control patients would tend to under-estimate any true year on year fluctuations. For example, if a quinquennium were to contain 4 years with 100 Parkinsonian births per year and a fifth with double this rate, then on average all 5 would be allocated 120 controls. Thus a true $100 \%$ increase over the background level would produce a P:C ratio of only $200: 120$, that is, a $66 \%$ increase. It was this ratio of 1.66 which was used in our definition of a cluster year. If Parkinsonian births do cluster as observed and if an external agent is at least partly responsible for the evolution of idiopathic Parkinson's disease, then the clustering allows two deductions to be drawn concerning the nature of such an agent. Firstly, it must act within a year of birth, and secondly its effects must fluctuate significantly from year to year such that individuals born in successive years are affected to differing degrees. Both these criteria must be fulfilled before clustering of patients by year of birth may be observed.

From the little known of developmental neural vulnerability the period of intra-uterine development could be a likely time for the action of an aetiological agent. Whilst it is possible for exposure to an environmental toxin to vary from year to year such waxing and waning would be in keeping with an infectious agent, particularly a virus or virus-like agent, which characteristically occurs in epidemics. The correspondence of the cluster years with the influenza pandemic suggests influenza as an obvious viral candidate. We therefore propose that intra-uterine influenza may predispose to the development of idiopathic Parkinson's disease in later life and this is supported by the positive correlation between relative idiopathic Parkinson's disease risk ( $\mathrm{P}: \mathrm{C}$ ratio) and influenzal activity $\log (\mathrm{A})$ for the years $1900-1930$. This correlation is moderately strong (coefficient $0.503, \mathrm{p}<0.005$ ). A much stronger correlation would be surprising as log (A) may be more a measure of influenzal respiratory toxicity, in a preantibiotic age, than of neurotoxicity. The seasonal variation with and idiopathic Parkinson's disease birth peak between March and June is compatible with intra-uterine influenza around 6 months gestation as the seasonal peak in influenzal activity is usually between January and March. ${ }^{7}$ Exprimental evidence suggests that respiratory toxicity is a common property of all influenzal strains but that neurotoxicity varies. ${ }^{8}$ The more neurotoxic strains interfere with sympathetic ganglionic transmission in both ferrets and cats. ${ }^{9}$

If our hypothesis is correct then there may be at 
least two contrasting sequelae following exposure of a foetal cohort to epidemic influenza. In one, the foetal cohort is exposed to an epidemic of mildly neurotoxic influenza. Each individual would sustain minor damage to the developing substantia nigra, according to his own susceptibility, but only a minority of individuals sustain sufficient initial nigral damage to precipitate clinical evidence of nigral failure in later life. Nigral failure may be delayed by many decades as cells are lost, and postnatal environmental exposures may modify their rate of decline and hence the interval before overt disease ensues. This model predicts that clinical idiopathic Parkinson's disease would develop in only a proportion of affected individuals after mildly neurotoxic influenza with presentation after a long latent interval, whilst the remainder with subclinical nigral damage die from other causes before nigral function reaches a critical level. In contrast, following an epidemic of a particularly neurotoxic influenza in which more extensive nigral damage occurred, allowing for individual variation, overt failure could occur in the majority of patients, susceptible individuals presenting after a short latent interval and perhaps before the age of 30 . In the years after the epidemic the age at presentation would fall to a mean of 30 to 40 years. Poskanzer and Schwab ${ }^{1}$ interpreted the year by year increase of onset of disease as representing a cohort phenomenon; they suggested that most patients had post-encephalitic disease, but this interpretation has not been generally accepted and does not adequately explain the presence of idiopathic Parkinson's disease before $1918,{ }^{1011}$ nor patients with idiopathic Parkinson's disease born after the epidemic of encephalitis.

An alternative explanation for the apparent cohort effect is that those born between 1889 and 1901 were exposed in utero to a particularly neurotoxic influenza which reached pandemic proportions in 1890-92 and caused epidemics in 1893, 1895 and 1898-1901, after 40 years of minimal influenzal activity (see fig 2).

Influenza has been tentatively linked with idiopathic Parkinson's disease since the 1920s when it was recognised that the post-infectious Parkinsonian syndrome could occur after an interval of weeks or several decades. Links between influenza $A$ and idiopathic Parkinson's disease have been sought. The evidence has been inconclusive, but does not exclude a role for influenza through the mechanism we propose. ${ }^{12}$ However, these data are unable to address the question of precise timing of infection which may cause enduring brain damage. Failure to find viral particles in Parkinsonian brains ${ }^{13}$ is significant, but influenzal infection very early in life cannot be excluded; in contrast to herpes viridae, influenza is not known to cause latent intracellular infection.

Apart from collecting more detailed information of the above kind, more direct tests of our hypothesis might be difficult. It would be unreliable to question
Parkinsonian patients whose parents are still alive and unrealistic to follow up the offspring of mothers who had reported influenza during pregnancy. Do other chronic and progressive neurological diseases of later life show a smoother distribution with respect to year of birth? Such data are lacking, but our controls gave no such indication. If short latency idiopathic Parkinson's disease in the 1930s was at least in part due to the prevalence of an unusually neurotoxic influenza in the 1890 s and intra-uterine infection, could history repeat itself? Unfortunately there is seroepidemiological evidence to suggest that the strain of 1890-92 pandemic may have already returned. Sera taken from elderly individuals before the influenza $A 2$ (Asian) pandemic of 1957 are reactive with antigens from both A2/1957 (Asian) strain and the A2 (Hong Kong) strain of the 1968 pandemic, suggesting that the same or a modified $1890-92$ strain was in currency in the 1960s. If these recent strains are neurotoxic and our model is correct then within a decade we may begin to see an increase in new presentations of idiopathic Parkinson's disease including younger patients and in particular those born in 1957-58 and 1968-69.

\section{References}

1 Poskanzer DC, Schwab RS. Cohort analysis of Parkinson's syndrome: evidence for a single aetiology related to subclinical infection about 1920.J Chron Dis 1963;16:961-73.

2 Godwin-Austen RB, Lee PN, Marmot MG, Stern GM. Smoking and Parkinson's disease. J Neurol Neurosurg Psychiatry 1982;45:577-81.

3 Stuart-Harris CH. Influenza. London: Edward Arnold, 1953.

4 Kilbourne ED, ed. The Influenza Viruses and Influenza. New York: Academic Press, 1975.

5 Hoyle L. The Influenza Viruses. Vienna: Springer-Verlag, 1968.

6 Registrar-General. Annual report of the RegistrarGeneral for England and Wales. Report nos 24-83. Annually 1861-1920. London: HMSO.

7 Registrar-General. The Registrar-General's statistical review of England and Wales. Annually 1921-1932. London: HMSO.

8 Stuart-Harris $\mathrm{CH}$. A neurotrophic strain of human influenza virus. Lancet 1939;i:497-9.

9 Ado AD, Alekseyeva TM. On the toxic effect of influenza virus on the sympathetic nervous system. Acta Virol 1957;1:161-6.

10 Kessler II. Parkinson's disease in epidemiological perspective. Adv Neurol 1978;19:355-83.

11 Duvoisin RC, Yahr MD, Schweitzer MD, Merritt HH. Parkinsonism before and since the epidemic of encephalitis lethargica. Arch Neurol 1963;9:232-6.

12 Martilla RJ, Halone P, Rinne UK. Influenza virus antibodies in Parkinsonism: comparison of postencephalitic and idiopathic Parkinson patients and matched controls. Arch Neurol 1977;34:99-100.

13 Schwartz J, Elizan TS. Search for viral particles and virus-specific products in idiopathic Parkinson's disease brains. Ann Neurol 1979;6:261-3. 